\title{
ON BIMODAL OFFSET CAUCHY DISTRIBUTION
}

\author{
S.V.S.GIRIJA, A.J.V.RADHIKA AND A.V.DATTATREYA RAO
}

\begin{abstract}
The bivariate Cauchy distribution has received applications in many areas, including biological analyses, clinical trials, stochastic modeling of decreasing failure rate life components, study of labour turnover, queuing theory and reliability (Nayak (1987) and Lee and Gross (1991)). In the study of biological analyses, clinical trials and reliability circular distributions will yield suitable results. Circular data arises in a number of different areas such as geological, meteorological, biological and industrial sciences. It is not suggestive to use standard statistical techniques to model circular data, due to the circular geometry of the sample space (p.2 Jammalamadaka and Sen Gupta (2001).

It is possible to construct a circular model by transforming a bivariate linear random variate to just its directional component and the resultant model is called 'offset distribution'. In the literature most of the available circular models were constructed by wrapping a linear model. In recent years some wrapped models were constructed by Dattatreya Rao et al (2007). Here an attempt is made to exploit method of offsetting on Bivariate Cauchy distribution to construct a circular model named by us "OFFSET CAUCHY DISTRIBUTION (OC)". The characteristic function of the Offset Cauchy model is derived and its characteristics are discussed.
\end{abstract}

Mathematics Subject Classification 2000: 60E05, 62H11

General Terms: Circular Models, Directional Data Analysis, Offsetting.

Additional Key Words and Phrases: Bivariate Linear Models, characteristic function, trigonometric moments, Fourier representation of Circular Model.

\section{INTRODUCTION}

The Most of the available circular models such as Circular Uniform, Wrapped Normal, Wrapped Cauchy etc. are derived by wrapping the respective linear models. By applying the same technique Girija (2010) constructed new wrapped models such as Wrapped Lognormal, Wrapped Logistic,Wrapped Weibull, Wrapped Extreme- value, Wrapped Halflogistic and Wrapped Binormal models.

Also a new method of construction called a family of the Rising Sun Circular models was introduced by Girija (2010). Since scant attention was paid for constructing offsetting models, here an attempt is made to construct a new circular model called “OFFSET CAUCHY DISTRIBUTION” by offsetting a Bivariate Cauchy distribution. 


\section{METHODOLOGY OF OFFSETTING}

A method of transforming a bivariate linear r.v. to its directional component is called OFFSETTING and the respective directional component is called offset distribution. This is done by accumulating probabilities over all different lengths for a given direction Transforming the bivariate random vector $(X, Y)$ into polar coordinates $(R, \theta)$ and integrate over $R$ for a given $\theta$ will yield a circular model ( Jammalamadaka and Sen Gupta (2001)) . If $f(x, y)$ denotes the joint density function, then the resulting circular offset distribution, say $g(\theta)$, is given by

$$
g(\theta)=\int_{0}^{\infty} f(r \cos \theta, r \sin \theta) r d r
$$

\section{CONSTRUCTION OF THE OFFSET CAUCHY DISTRIBUTION}

The Offset Cauchy (OC) distribution is derived from the Bivariate Cauchy distribution $f(x, y)$. Balakrishnan and Chin (2008) have given the joint density function of Bivariate Cauchy distribution as

$$
f(x, y)=\frac{1}{2 \pi \sigma_{1} \sigma_{2}}\left(1+\frac{x^{2}}{\sigma_{1}^{2}}+\frac{y^{2}}{\sigma_{2}^{2}}\right)^{-3 / 2} \text { where } x, y \in, \sigma_{1}>\sigma_{2}>0
$$

Using the technique explained in section (2), the probability density function of the Offset Cauchy distribution (OC) is derived as under

$$
\begin{aligned}
g(\theta) & =\int_{0}^{\infty} f(r \cos \theta, r \sin \theta) r d r \\
& =\int_{0}^{\infty} \frac{1}{2 \pi \sigma_{1} \sigma_{2}}\left(1+\frac{r^{2} \cos ^{2} \theta}{\sigma_{1}^{2}}+\frac{r^{2} \sin ^{2} \theta}{\sigma_{2}^{2}}\right)^{-3 / 2} r d r,\left(\text { put } r^{2}=t\right) \\
& =\int_{0}^{\infty} \frac{1}{2 \pi \sigma_{1} \sigma_{2}}\left(1+\left(\frac{\cos ^{2} \theta}{\sigma_{1}^{2}}+\frac{\sin ^{2} \theta}{\sigma_{2}^{2}}\right) t\right)^{-3 / 2} \frac{d t}{2} \\
& =\frac{\sigma_{1} \sigma_{2}}{2 \pi\left(\sigma_{2}^{2} \cos ^{2} \theta+\sigma_{1}^{2} \sin ^{2} \theta\right)} \text { where } \sigma_{1}>\sigma_{2}>0, \theta \in[0,2 \pi)
\end{aligned}
$$

This function satisfies the properties of circular model viz., in the continuous case 


\section{ON BIMODAL OFFSET CAUCHY DISTRIBUTION}

$g:[0,2 \pi) \rightarrow \mathbb{R}$ is the probability density function of a circular distribution iff $g$ has the following basic properties

- $\quad g(\theta) \geq 0, \quad \forall \theta$

- $\quad \int_{0}^{2 \pi} g(\theta) d \theta=1$

- $\quad g(\theta)=g(\theta+2 k \pi)$ for any integer $k$ (i.e., $g$ is periodic) Mardia (1972)

Hence the function (3) represents a probability density function of a circular model, the Offset Cauchy distribution. When $\sigma_{1}=\sigma_{2}$, the density reduces to the uniform density. The cumulative distribution function is given by

$$
\begin{aligned}
G(\theta) & =\int_{0}^{\theta} g(\alpha) d \alpha=\int_{0}^{\theta} \frac{\sigma_{1} \sigma_{2}}{2 \pi\left(\sigma_{2}^{2} \cos ^{2} \alpha+\sigma_{1}^{2} \sin ^{2} \alpha\right)} d \alpha \\
& \left.=\frac{\sigma_{1} \sigma_{2}}{2 \pi} \int_{0}^{\tan \theta} \frac{d t}{\left(\sigma_{2}^{2}+\sigma_{1}^{2} t^{2}\right)} \quad \text { (put } \tan \alpha=\mathrm{t}\right) \\
& = \begin{cases}\frac{1}{2 \pi} \tan ^{-1}\left(\frac{\tan \theta}{\sigma_{2} / \sigma_{1}}\right) & \text { for } 0 \leq \theta<\frac{\pi}{2} \\
\frac{1}{2 \pi}\left\{\pi+\tan ^{-1}\left(\frac{\tan \theta}{\sigma_{2} / \sigma_{1}}\right)\right\} & \text { for } \frac{\pi}{2}<\theta<\frac{3 \pi}{2} \\
\frac{1}{2 \pi}\left\{2 \pi+\tan ^{-1}\left(\frac{\tan \theta}{\sigma_{2} / \sigma_{1}}\right)\right\} & \text { for } \frac{3 \pi}{2}<\theta<2 \pi\end{cases}
\end{aligned}
$$

On the lines of Ramabhadra Sarma et al (2009), (2011) the characteristic function of the Offset Cauchy distribution is derived. By applying the Cauchy Residue Theorem the characteristic function of the Offset Cauchy distribution is obtained as 


$$
\begin{aligned}
\varphi_{\theta}(p) & =\int_{0}^{2 \pi} e^{i p \theta} \frac{\sigma_{1} \sigma_{2}}{2 \pi\left(\sigma_{2}^{2} \cos ^{2} \theta+\sigma_{1}^{2} \sin ^{2} \theta\right)} d \theta, p \in \\
& =\frac{a^{p}\left(1+(-1)^{p}\right)}{2} \quad \text { where } a=\sqrt{\frac{\sigma_{1}-\sigma_{2}}{\sigma_{1}+\sigma_{2}}}
\end{aligned}
$$

The Fourier representation of the pdf of the circular model is [Jammalamadaka and Sen Gupta (2001)]

$$
\begin{aligned}
g(\theta) & =\frac{1}{2 \pi} \sum_{-\infty}^{\infty} \phi_{p} e^{-i p \theta} \\
& =\frac{1}{2 \pi}\left[1+2 \sum_{p=1}^{\infty}\left(\alpha_{p} \cos p \theta+\beta_{p} \sin p \theta\right)\right] \theta \in[0,2 \pi), p \in \mathbb{Z}
\end{aligned}
$$

where the convergence of the sum is in the $\mathrm{L}^{2}$ sense.

Here in the case of Offset Cauchy model it is shown as

$$
\begin{aligned}
g(\theta) & =\frac{1}{2 \pi}\left[1+2 \sum_{p=1}^{\infty} a^{p} \cos p \theta\right] \\
& =\frac{1}{2 \pi}\left[1+2 \sum_{k=1}^{\infty} a^{2 k} \cos 2 k \theta\right] \quad \text { where } a=\sqrt{\frac{\sigma_{1}-\sigma_{2}}{\sigma_{1}+\sigma_{2}}} \\
& =\frac{1}{\pi}\left[\sum_{k=0}^{\infty} a^{2 k} \cos 2 k \theta-\frac{1}{2}\right]
\end{aligned}
$$




\section{GRAPH AND THE CHARACTERISTICS OF THE MODEL}

The linear representation of the graph of the pdf of the Offset Cauchy distribution for various values of $\sigma_{1}$ and $\sigma_{2}$ can be plotted using MATLAB.

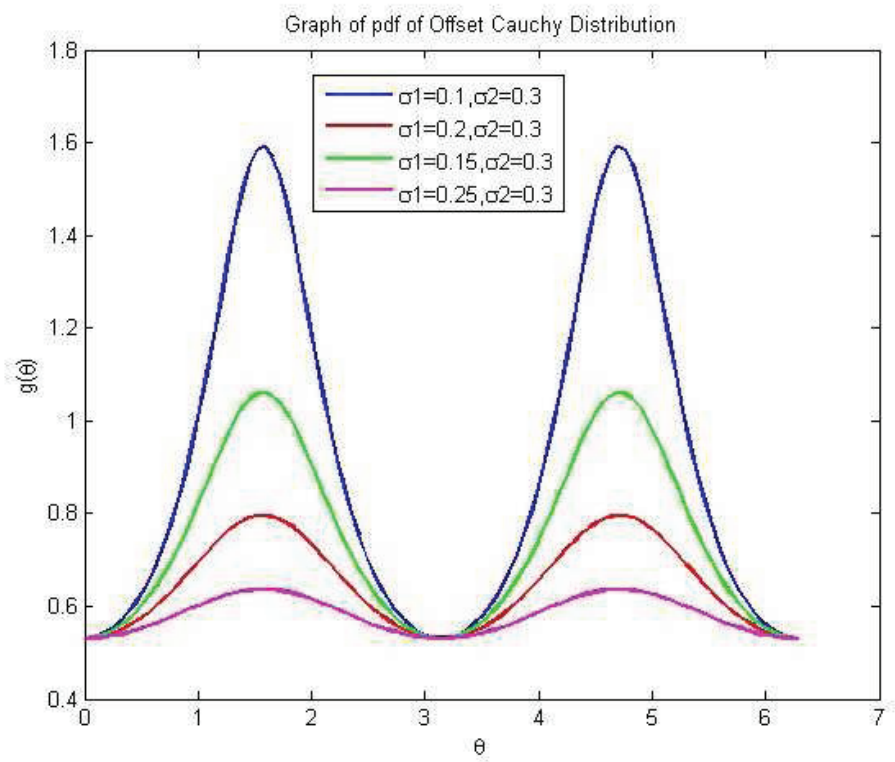

Fig. 1. Pdf of the Offset Cauchy Distribution

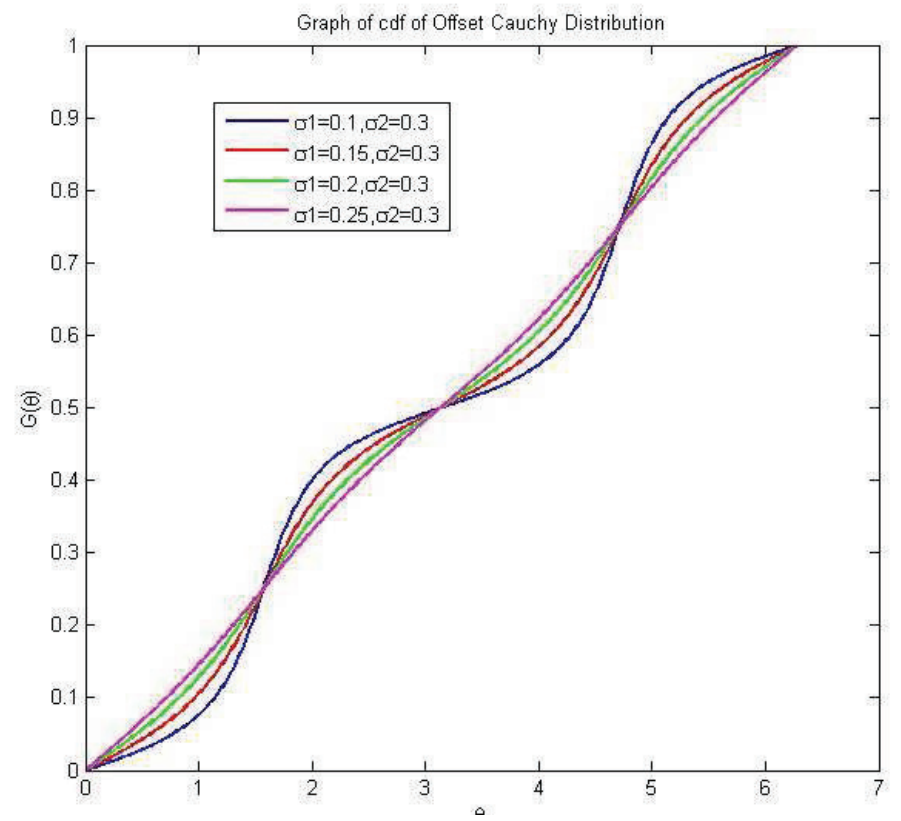

Fig. 2. Cdf of the Offset Cauchy Distribution 


\section{S.V.S.GIRIJA, A.J.V.RADHIKA, A.V.DATTATREYA RAO}

It is observed that the density of the Offset Cauchy distribution is bimodal only if $\sigma_{1}$ and $\sigma_{2}$ are unequal and it is symmetric distribution. The value of the characteristic function at an integer $p$ is also called the $p^{\text {th }}$ trigonometric moment of $\theta$. Here first trigonometric moments $\alpha_{1}$ and $\beta_{1}$ are zeros. Hence properties of the distribution can't be evaluated by the formulae available in Mardia (2000). The mean is defined as zero and the variance is one.

\section{ACKNOWLEDGMENTS}

Authors would like to thank UGC, New Delhi, India for offering financial assistance to carry out the project under the head of Major Research Project no. F 41-785/2012 (SR) dt. 17-07-2012.

\section{REFERENCES}

BALAKRISHNAN, N. AND CHIN - DIEW LAI (2008), Continuous Bivariate Distributions Springer.

BATCHELET,E. (1981), Circular Statistics in Biology, Academic Press, London.

BRECKLING, J. (1989), The Analysis of Directional Time Series: Applications to Wind Speed and Direction, Springer Verlag, New York.

DATTATREYA RAO, A.V., RAMABHADRA SARMA, I. \& GIRIJA, S.V.S. (2007) On Wrapped Version of Some Life Testing Models, to appear in Communication in Statistics-Theory and Methods , 36,\# issue 11.

GIRIJA, S.V.S. (2010), Construction of New Circular Models ,ISBN 978-3-639-27939-9 VDM Verlag Dr. Müller GmbH \& Co. KG.

HANBURY, A. 2003, Circular statistics applied to colour images. 8th ComputerVision Winter Workshop. HRUSHESKY, W. J. M., (1994), Circadian Cancer Therapy, CRC Press, Boca Raton.

JAMMALAMADAKA S. RAO \& SEN GUPTA, S. (1972), Mathematical Techniques for Paleocurrent Analysis: Treatment of Directional Data, Journal of intl Assoc. Geol, 4, 235 - 258.

JAMMALAMADAKA S. RAO \& SEN GUPTA, A. (2001), Topics in Circular Statistics, World Scientific Press, Singapore.

KENDALL, D.G. (1974), Pole - Seeking Brownian Motion and bird navigation, J. Roy. Statist. Soc., 36, 365 417.

LEE, M.L.T. AND GROSS, A.J. (1991), Lifetime distributions under unknown environment, J. Statist. Plann. Inference 29, 137-143.

MARDIA, K.V. (1972), Statistics of Directional Data, Academic Press, NewYork.

MARDIA, K.V. \& JUPP, P.E. (2000), Directional Statistics, John Wiley, Chichester.

MORGAN, E., (1990), Chronobiology and Chronomedicine, Peter Lang, Frankfurt.

NAYAK, T.K. (1987), Multivariate lomax distribution - Properties and usefulness in reliability theory, J. Appl. Probab. 24, 170-177.

RAMABHADRA SARMA, I. DATTATREYA RAO A.V. AND GIRIJA, S.V.S. (2009), On Characteristic Functions of Wrapped Half Logistic and Binormal Distributions, International Journal of Statistics and Systems, Volume 4 Number 1, pp. 33-45.

RAMABHADRA SARMA, I. DATTATREYA RAO A.V. AND GIRIJA, S.V.S. (2011) On Characteristic Functions of Wrapped Lognormal and Weibull Distributions, Journal of Statistical Computation and Simulation Vol. 81, No. 5, 579-589.

RAO, J.S., BHADRA, N., CHATURVEDI, D., KUTTY, T.K., MAJUMDAR, P.P., AND

PODUVAL, G. (1986), Functional assessment of knee and ankle during level walking, Data Analysis in Life Science, 21 - 54, Indian Statistical Institute, Calcutta, India.

SCHMIDT - KOEING, K. (1963), On the role of loft, the distance and site of release in Pigeon homing (the "cross - loft experiment "), Biol. Bull., 125, 154- 164. 
ON BIMODAL OFFSET CAUCHY DISTRIBUTION

S V S Girija,

Associate Professor of Mathematics,

Hindu College, Guntur,

India

Email : svs.girija@gmail.com

A J V Radhika,

Assistant Professor of Mathematics,

ANU College of Engineering,

Acharya Nagarjuna University,

India

Email: ajv.radhika09@gmail.com

A V Dattatreya Rao,

Professor of Statistics,

Acharya Nagarjuna University,

India

Email: avdrao@gmail.com

Received May 2013 